His co-author is Ann Westmore, a medical journalist whose claim to fame is apparently a best-selling birth control book on The Billings Method. The book first tackles the process of conception and answers basic questions about infertility. Then there is a chapter about the development of the test-tube baby programme in Melbourne. The next few chapters describe the sort of patients who can be helped and go into the minutiae of the treatment and the stresses and practical problems, written very much with the patient in mind. For the few successful patients there is a reassuring chapter on the outcome of pregnancy, but this is put into perspective by a subsequent chapter on the limited current success of the procedure. Following this there is an ethical, social and religious justification of the procedure, written in the question and answer style. Although this chapter, on initial reading, comes across as rather complacent and glib, it has to be kept in mind that the book is aimed at neither the informed physician nor the moral philosopher. The final chapter on future prospects launches bravely into cloning and sperm microinjection as well as some of the other biological scenarios that have been perpetrated in Melbourne such as embryo donation, and makes rather too little of the less sensational, but more relevant advances in knowledge that can be achieved with continuing embryo research.

All in all, this is a book that has been written for the lay person rather than the professional, and I shall certainly be recommending it to my patients. Possibly I should have asked one of them to review it.

ALLAN TEMPLETON Department of Obstetrics and Gynaecology, University of Edinburgh

\section{A Guide to Symptom Relief in Advanced Cancer}

\author{
Regnard C F B, Davies A, Randall F. 44 \\ pages, Manchester, $£ 2.95$, Haigh and \\ Hochland, 1983.
}

The aim of this short book is to provide basic guidelines for the control of symptoms of patients dying of cancer. It is primarily concerned with therapeutics and ethical issues are not considered. Pain control dominates the first part. The common reasons for failure to control pain are considered: fear of analgesics, failure to diagnose, lack of pharmacological knowledge, poor use of adjuvant medication and inadequate reassessment. These common fears are allayed as the use of oral medication for pain control is discussed. The very limited need for injections is clearly stressed. Adjuvant medication to opiate analgesia is explained, but the relevant table appears too early in the chapter and anticonvulsants are not mentioned.

Diagnosis of the cause of the pain is not sufficiently emphasised. Correct treatment depends upon accurate diagnosis and most patients have several pains, each of which needs to be assessed. Some pains may be unrelated to the cancer and require different treatment.

A clear methodical approach of diagnosis leading to treatment is introduced when the control of nausea and vomiting is considered and there is a useful table of anti-emetic drugs. However, this approach is not always maintained with other symptoms and the need to diagnose is neglected in the enthusiasm to discuss treatment. Certain important and distressing symptoms, such as dyspnoea, are rather scantily covered. There is a very useful table of drug interactions and a full list of references.

However, the information is often lost in a complete jumble of headings and sub-headings, leaving the reader easily confused. Although this book could be helpful to many doctors, the presentation may in fact limit its usefulness.

D J OLIVER

Medical Director, The Wisdom Hospice, Rochester and Consultant Physician in Continuing Care to the Medway Health Authority

\section{Freud and Human Nature}

Dilman I. 207 pages, Oxford, £15, Blackwell, 1983

\section{Freud and the Mind}

Dilman I. 204 pages, Oxford, £15, Blackwell, 1983.

These clearly written and extensive investigations into the philosophical underpinnings of Freud's psychology are part of a larger research programme in which Dilman attempts a reconstruction, rather than an exegesis, of Freudian theory. Freud and Human Nature differs from most other studies on Freud in itso criticism of his hedonistic conception oft sexuality, of his account of the relationship between love and sexuality, ? and of his polarisation of human nature and culture and of instinct and morality It might be expected that when theseo theories have been removed there is littleo else of interest in Freud's programme. $\overline{\bar{p}}$. For Dilman, however, it is a necessary step towards seeing what is of lasting importance in Freud.

In the first two chapters of Freud and $\overrightarrow{0}$ Human Nature Dilman argues that even on Freud's terms a purely hedonistic $\vec{\omega}$ sexuality is really an infantile sexuality. $\stackrel{S}{S}$ Although the lifting of sexual repression $\overline{3}$ was important to Freud, it should not, $₫$ insists Dilman, be seen as either an $\vec{\overrightarrow{ }}$ advocacy of 'giving way to impulse' or as î a means of removing guilt in order to take $\vec{\circ}$ an easy way out of moral obligations. In the following chapters Dilman's critique is expanded into a reappraisal of Freud's 군 negativistic account of morality and his view of culture as an external restraint on $\subseteq$ instinct and human nature. In the course of his rejection of the quasi-mechanistic $\rightarrow$ polarities which dominate Freud's $\odot$ writings Dilman argues that Freud 8 s or only acceptable as a psychologist gif purged from his philosophim presuppositions. But this raises mam problems than Dilman appears to recognise. It may be the case that bad philosophy distorts a scientific $\mathbb{Q}$ programme, but the remedy lies in its $\vec{\overrightarrow{ }}$ replacement with a better and more $\frac{O}{3}$ rigorous philosophical content. Yet $\mathcal{F}$ Dilman conveys the impression that it is the very contact with philosophy - rather than bad philosophy - which distorts Freud's psychology. Thus Freud? encounters 'certain philosophical 3 difficulties' and suffers from'philosophical confusions' whilst $\frac{O}{2}$ 'philosophical froth' and 'philosophical presuppositions' tend to 'get in the way'음 and 'distort his insight'. In this respect $>$ Dilman shares an assumption - 을 apparently held by Wittgenstein and Rhees - that the shedding of any o philosophical content is essential to N scientific progress. This kind of philosophic aversion therapy may be a $\mathrm{\omega}$ useful prophylactic against the wilder speculations of philosophers but it cane lead to a narrow and distortive $\bar{\varnothing}$ interpretation of the sciences and their? requirement for a sound appraisal of their $\bar{T}$ conceptual apparatus, which is ${ }_{0}$

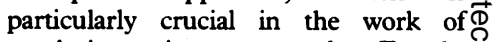
revolutionary innovators such as Freud. $\overrightarrow{\mathbb{D}}$

There is little doubt that Dilman has $\cong$ great respect for Freud and there is considerable value in this reconstruction 
of his work. However, the chapter on love and sexuality tends to ramble and relies perhaps too heavily on Wittgenstein, Rhees, and literary sources to establish a fairly obvious point that love is a many splendoured thing, and that sexual love involves more than desire. All of this detracts from an otherwise scholarly critique which is developed out of an excellent appraisal of Malinkowski, Klein and Reich.

Whereas Freud and Human Nature concentrates on Freud's views on the role of sexuality in human life, morality, and the relationship between the individual and society, Freud and the Mind is concerned with his account of the unconscious, phantasy, emotions, repression, and free will. Here it is argued that Freud's mechanistic and causal language prevents a full appreciation of his achievements and that his philosophically-based determinism conflicts with his intention to liberate the analysand. Here too Dilman's objective is to remove the 'philosophical froth' from Freud's theory. We find that Freud's determinism is philosophical excess baggage: although he made an original and lasting contribution to psychology 'the language in which he presents his contribution reflects the philosophical prejudices of his time'. Again, it is philosophy that prevents Freud from saying what he wants to say, and it is the philosophy embedded in the popular versions of Freud in the contemporary consciousness that robs his work of any depth. Dilman does not attempt to equip Freudian theory with a more acceptable philosophical framework; his aim is simply to purge the theory from philosophical contamination.

The obvious merit of Freud and the Mind lies in the way Dilman traces the development of Freud's discovery of the unconscious, explores the possible meanings of unconscious memories and phantasies, and examines the extent to which the unconscious can be said to determine thought and action. Nevertheless, it is very hard to assess many of the arguments. Unlike Freud and Human Nature, where Malinkowski's anthropological work is frequently cited, this text suffers from a scarcity of scientific sources. Not a single psychiatric case study is cited. There are no references to recent empirical research, no treatment of recent discussions in the journals of psychiatry. Instead the critique of Freud is conducted with reference to a range of literary characters in the works of Shakespeare, Proust, Dostoyevsky, and Tolstoy. A preference for literary studies over case studies might provide an acceptable mode of philosophical inquiry in some quarters but the value of deriving clinical criteria from literary insights is highly dubious. Psychologists and psycho-analysts would be ill-advised to base their diagnoses on an analysis of Crime and Punishment or recommend a course of therapy on the basis of insights derived from an account of Kitty's selfdeception in Anna Karenina. The attempt to rid Freud of 'philosophical froth' ultimately involves the replacement of Freud's philosophical presuppositions with those held by fiction writers. Dilman has attempted a bold and original critique of the philosophical underpinnings of psychoanalysis, but his almost exclusive reliance upon fiction and an unquestioned appeal to the authority of Wittgenstein, Weil, Rhees, suggests that the book is of greater value to the literary critic than the clinical therapist.

DAVID LAMB Lecturer in Philosophy, University of Manchester

\section{The Influence of Christians in Medicine}

Editors, J T Aitken, H W C Fuller and D Johnson, 186 pages, London, $£ 3.95$, Christian Medical Fellowship, 157 Waterloo Rd, SE1 8XN, 1984

Thirteen of the chapters in this book appear to have been written cooperatively by the editors, and one, on 'The Spread of Western Medicine', is by Gordon A D Lavy. The book is designed primarily for Christian medical folk of an evangelical outlook, in the sense that after the Reformation period it concentrates on 'Protestant medical circles. But it is in no sense a partisan work. It is written in an eirenic spirit, and makes full allowance, among other matters, for the contribution of Arabs and Jews in the development of medicine. Since we tend to be preoccupied with the issues and controversies of our own time it is wise to achieve a sense of perspective by reflecting on how we came to where we are, and this is true of Christians and the Christian tradition in medicine.

This book is narrative rather than analytical history, fitting the contribution of outstanding individuals into an historical framework. Henceforth, it is remarked, this will no longer be possible because increased technical complexities have made medicine much more a corporate activity. How a Christian contribution can best be made in these circumstances is beyond the scope of the book. There are hints of a polarisation between Christianity and what is incidentally referred to as 'agnostic', 'atheist' or 'scientific' humanism which could make such a contribution difficult. If the hints were developed they would probably over-simplify the polarity. But parallel to this is the contention that monotheism is a stimulus to medicine because it means we view the world as orderly, and not as subject to the caprice of pagan deities or mere chance. The picture emerges of a long line of devoted individual practitioners, often embroiled with a Church frequently in error, sometimes corrupt, and inclined to be jealous. The defects of the individuals concerned are by comparison underplayed. Physicians and surgeons naturally are the focus of attention, but in modern times representatives of nursing and preventative medicine are brought into the narrative. There are no specific references, but details of the chief sources consulted and an index of all the persons referred to, as well as a subject index, are provided. The book deliberately excludes psychological medicine, as being too large a field, and 'faith healing' because its concern is with 'orthodox medicine'. If there are any remnants of the idea around that Christianity has been hostile to the latter down the centuries (an idea propagated by secularist movements earlier in this century), this book will scotch them. Of modest size, this book may well be of interest to others beyond the Christian Medical Fellowship for whom it was produced.

RONALD PRESTON Emeritus Professor of Social and Pastoral Theology, Manchester University

\section{Professionalism and the Empowerment of Nursing}

American Nurses' Association, 76 pages, Missouri, American Nurses Association, 1982.

This is a collection of papers concerned with the professional status of nursing and, more importantly, with the power which goes along with that status. The papers were presented at the 53rd convention of the American Nurses' Association in 1982. According to the preface the papers in this volume 'show the determination of nurses to take the 